\title{
CrowdLearn: Crowd-sourcing the Creation of Highly-structured e-Learning Content
}

\author{
http://dx.doi.org/10.3991/ijep.v5i4.4951 \\ D. Tarasowa ${ }^{1}$, A. Khalili ${ }^{2}$ and S. Auer ${ }^{1}$ \\ ${ }^{1}$ Rheinische Friedrich-Wilhelms-Universität Bonn, Germany \\ ${ }^{2}$ Vrije Universiteit Amsterdam, The Netherlands
}

\begin{abstract}
While nowadays there is a plethora of Learning Content Management Systems, the collaborative, community-based creation of rich e-learning content is still not sufficiently well supported. Few attempts have been made to apply crowd-sourcing and wiki-approaches for the creation of e-learning content. However, the paradigm is only applied to unstructured, textual content and cannot be used in SCORM-compliant systems. To address this issue we developed the CrowdLearn concept to exploit the wisdom, creativity and productivity of the crowd for the creation of rich, deep-semantically structured e-learning content. The CrowdLearn concept combines the wiki style for collaborative content authoring with SCORM requirements for reusability. Therefore, it enables splitting the learning material into Learning Objects (LOs) with an adjustable level of granularity. In order to realize the CrowdLearn concept, a novel data model called WikiApp is devised. The WikiApp data model is a refinement of the traditional entityrelationship data model with further emphasis on collaborative social activities and structured content authoring. We implement and evaluate the CrowdLearn approach with SlideWiki - an educational platform dealing with presentations and assessment tests. The article also comprises results of a usability evaluation with real students..
\end{abstract}

Index Terms-crowdsourcing, e-learning, LMS, structured learning objects, SCORM.

\section{INTRODUCTION}

While nowadays there is a plethora of Learning Content Management Systems (LCMS), the collaborative, community-based creation of rich e-learning content is still not sufficiently well supported. Few attempts have been made to apply crowd-sourcing and wiki-approaches for the creation of e-learning content. Wikiversity ${ }^{1}$, for example, is a Wikimedia Foundation project aiming to leverage standard wiki technology for the creation of hypertext e-learning content. Peer 2 Peer University $(P 2 P U)^{2}$ and PlanetMath $^{3}$ are other examples which employ crowdsourcing to create rich e-learning content. P2PU helps users to navigate the wealth of open education materials and supports the design and facilitation of courses. The PlanetMath is a project to aiming to become a central repository for mathematical knowledge on the web, with a pedagogical mission. However, we deem, that no real attempt has been made so far to truly apply the concepts

\footnotetext{
' http://wikiversity.org

2 http://p2pu.org/

${ }^{3}$ http://planetmath.org/
}

behind wikis and crowd-sourcing to develop a specifically tailored technology supporting the creation of highlystructured, SCORM-compliant e-learning content.

\section{Sharable Content Object Reference Model} (SCORM) [1] as one of the community-approved standards, requires the transformation of the learning material into the sequence of annotated Sharable Content Objects (SCOs). The granularity and sequencing of the SCOs should be determined by the content author depending on the audience needs and preferences [2] Ward Cunningham's wiki [10] paradigm is mainly only applied to unstructured, textual content. This limitation makes it difficult or even impossible to use the wiki style of content authoring in the SCORM-compliant learning platforms. As a result, a proper community collaboration, authoring, versioning, branching, reuse and re-purposing of (semi)structured educational content similarly as we know it from the open-source software community is currently not supported. To address the issue we develop the CrowdLearn concept.

CrowdLearn exploits the wisdom, creativity and productivity of the crowd for the creation of rich, deepsemantically structured e-learning content. It combines the wiki style of collaborative content authoring with SCORM requirements for re-usability. Therefore, it enables splitting the learning material into Learning Objects (LOs) with an adjustable level of granularity. The CrowdLearn concept is based on five fundamental components (cf. Section III): standard compliance, semantic structuring, enhanced possibilities for reuse and re-purpose, crowdsourcing and social networking.

In order to implement and evaluate the CrowdLearn concept, we created a showcase application named SlideWiki. SlideWiki deals with the collaborative creation of original e-learning content such as presentations, slides, diagrams and self-assessment tests. During the implementation of the CrowdLearn concept, we faced several challenges (cf. Section IV). For enabling the high-level collaboration, all content should be versioned, similar to the wiki paradigm. SCORM has direct support for multipleversion content objects. However, we needed rules for triggering the creation of new revisions. Our findings on this issue are presented in sec:revisions. The next challenge was the SCORM requirement for the content to be structured. To solve this we developed the WikiApp data model [15] that organizes the relations between different content objects. Finally, the third challenge was to involve the learners in the process of content creation. We addressed this issue by providing support for social networking activities. Both content owners and students are able to 
participate in discussions about the learning material. While SCORM allows content engineers to do the sequencing, we allow it to be done by learners as well. As a consequence, semantically structured learning objects can be created and edited in a truly collaborative way. We further evaluated the CrowdLearn concept by conducting a comprehensive usability study with real students using SlideWiki (cf. Section V).

\section{RELATED WORK}

Related work can be roughly divided into the following two categories:

\section{A. Collaborative creation of e-learning content.}

The importance of creating reusable and re-purposable e-learning objects is widely accepted by the e-learning community [5]. However, most of the works address the learning object reuse problem rather by means of semantic meta-data annotations, content tagging and packaging than by creating richly structured, reusable learning objects from the ground. The importance of creating learning objects already with reuse in mind was, for example, stated by [13]: Content ... should be represented not as an object of study but rather as necessary elements towards a series of objectives that will be discovered in the course of various tests. There are only few approaches for the direct authoring of reusable content, such as, for example, learning examples creation [9] or semantic structuring and annotation of video fragments [4].

We should also mention the Learning Objects Repositories (LORs), that allow to produce structured reusable content. The first of them, Connexions ${ }^{4}$, presents the learning material as a combination of paragraphs, each of them could be easily edited or deleted. However, this structuring is done more for comfortable editing and does not have any functional benefits: the paragraphs cannot be reused or annotated independently. Thus, Connexions presents just an improved user interface for wiki-based system. The second example of structuring, that is more close to our idea, is LeMill ${ }^{5}$. LeMill provides a way of collaborative editing of the presentations by implementing presentations as a group of images which can be edited collaboratively. However, to edit a slide, a user has to replace it with another one. Also, it is impossible to have several subgroups of the slides within a presentation. The search through the slides (not presentations) is also not implemented. Thus, slides cannot be manipulated as independent learning objects.

The CrowdLearn concept differs from the existing approaches for managing e-learning content. It enables the construction of semantically structured learning objects from existing sources by combining, reordering and simple editing. By the term semantically structured object we mean that all the parts within the structure own all the attributes and methods of the object type, or, in the case of learning content, are complete and fully-functional LOs.

\section{B. Wiki-based collaborative knowledge engineering.}

The importance of wikis for collaborative knowledge engineering is widely acknowledged. In [14], for example, a knowledge engineering approach which offers wiki-style collaboration, is introduced aiming to facilitate the capture

\footnotetext{
${ }^{4}$ http://cnx.org/
}

${ }^{5}$ http://lemill.net/ of knowledge-in-action which spans both explicit and tacit knowledge types. The approach extends a combined rule and case-based knowledge acquisition technique known as Multiple Classification Ripple Down Rules to allow multiple users to collaboratively view, define and refine a knowledge base over time and space. In a more applied context, [6] introduces the concept of wiki templates that allow end-users to define the structure and appearance of a wiki page in order to facilitate the authoring of structured wiki pages. Similarly the hybrid wiki approach [12] aims to solve the problem of using (semi-)structured data in wikis by means of page attributes. In our approach we apply the wiki paradigm to the creation and collaboration around (semi-)structured learning objects.

\section{CROWDLEARN CONCEPT}

We see the CrowdLearn concept as an application of crowd-sourcing techniques to the e-learning content creation, re-purpose and reuse. As shown in Figure 1, the concept is based on the following five strategies:

\section{A. Standard-compliance}

The costs associated with building high-quality elearning content are high. One solution to decrease the costs is to author structured and reusable e-learning content that can be repurposed in different ways. To facilitate this, it should be possible to migrate content between different Learning Management Systems (LMSs). However, often content migration is not completely adequate and can thus result in loss of valuable content, meta-data or structure. Even if the transfer is possible, moving the content between systems can be more costly than just redeveloping that course in the new system. The strategy to overcome this challenge is the standard-compliance of both LMS and content. In that regard, we adopted the SCORM standard [1] and practical recommendations [2] and expanded the standard for the collaborative model.

\section{B. Semantic structuring}

Instead of dealing with large learning objects (often whole presentations or tests), we decompose them into fine-grained learning artifacts. Thus, rather than a large presentation, user will be able to edit, discuss and reuse individual slides; instead of a whole test she/he will be able to work on the level of individual questions. This concept efficiently facilitates the reuse and re-purpose of the learning objects. To implement the concept, we employ the WikiApp approach, as discussed in [15].

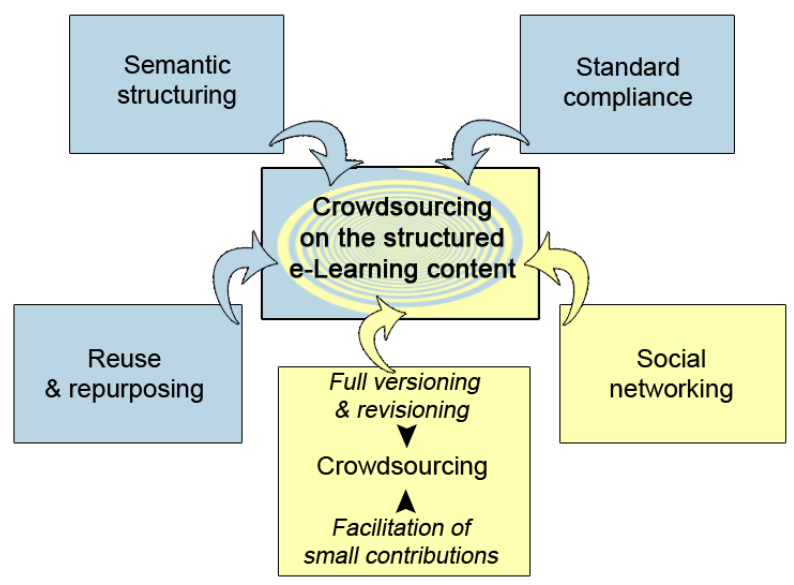

Figure 1. CrowdLearn concept. 


\section{Reuse and re-purpose}

The benefits of reuse and repurposing are: (1) increasing the cost efficiency of content creation, (2) increasing the quality of e-learning content and (3) supporting the evolution and adaptation to new requirements. To increase the economic efficiency of e-learning, content should be reused for a long period of time. Then, the development costs can be amortized over several years. However, the student expectations for higher quality e-learning experience increase, and new technology emerges so quickly that most courses need redeveloping every 3-4 year [8]. Instead of the full redevelopment, the content can slightly evolve. In that case the courses remain competitive with regard to the provisioning of high quality e-learning content. The possibility to reuse and re-purpose is crucial for the e-learning content evolution. Also, re-purposing allows to increase the efficiency by teaching more learners with the same content.

\section{Crowd-sourcing}

There are already vast amounts of amateur and expert users which are collaborating and contributing on the Social Web. Harnessing the power of such crowds can significantly enhance and widen the distribution of elearning content. Crowd-sourcing as a distributed problem-solving and production model is defined to address this aspect of collective intelligence [7]. CrowdLearn as its main innovation combines the crowd-sourcing techniques with the creation of highly-structured e-learning content. E-learning material when combined with crowdsourcing and collaborative social approaches can help to cultivate innovation by collecting and expressing (contradicting) individual's ideas. As Paulo Freire wrote in his 1968 book Pedagogy of the Oppressed, 'Education must begin with the solution of the teacher-student contradiction, by reconciling the poles of the contradiction so that both are simultaneously teachers and students...'. Therefore, crowd-sourcing in the domain of educational material not only increases the amount of e-learning content but also improves the quality of the content.

\section{E. Social networking}

The theoretical foundations for e-Learning 2.0 are drawn from social constructivism [19]. It is assumed that students learn as they work together to understand their experiences and create meaning. In this view, teachers are knowers who craft a curriculum to support a self-directed, collaborative search and discussion for meanings. Supporting social networking activities in CrowdLearn enables students to proactively interact with each other to acquire knowledge. With the CrowdLearn concept we address the following social networking activities:

- Users can follow individual learning objects as well as other users activities to receive notification messages about their updates.

- Users can discuss the content of learning objects in a forum-like manner.

- Users can share the learning objects within their social network websites such as Facebook, Google Plus, LinkedIn, etc.

- Users can rate the available questions in terms of their difficulty.

Besides increasing of the learning process quality, social activities improve the quality of the created learning material. Even when answering a quiz, users can contribute by analyzing the quality of the questions and making suggestions of how to improve them. Thus, the knowledge is being created not only explicitly by contributors, but also implicitly through discussions, answering the questions of assessment tests, or in other words through native learning activities.

\section{CROWDLEARN IMPLEMENTATION}

We implement and evaluate the CrowdLearn concept with SlideWiki ${ }^{6}$ - a web-based crowd-learning platform. SlideWiki deals with two types of (semi-)structured learning objects: slide presentations and assessment tests. SlideWiki follows our proposed WikiApp data model [13] to facilitate the creation, re-purpose and reuse of learning objects. Due to the space limit we do not elaborate on the WikiApp data model in the current paper and focus on the implementation details instead.

In order to be able to evaluate the future implementation of the CrowdLearn concept we at first conducted a systematic literature study [18]. In the scope of the study we have included the research papers for the latest ten years addressing different aspects of collaborative authoring of educational content. During the research we have defined three main aspects of the collaborative aithoring: support of collaboration (1), support of content reuse and re-purpose (2) and support of social aspects (3). These three aspects include further issues (see Table I). In the study we collected and compared the approaches for solving the issues. In the current Section we discuss the SlideWiki solutions and implementation for the issues.

\section{A. Collaborative authoring of content and metadata}

SlideWiki employs an inline HTML5 based WYSIWYG (What-You-See-Is-What-You-Get) text editor for authoring the presentation slides (cf. Figure 2, image 1). Using this approach, users will see the slideshow output at the same time as they are authoring their slides. The editor is implemented based on ALOHA editor $^{7}$ extended with some additional features such as image manager, source manager, equation editor. The inline editor uses SVG images for drawing shapes on slide canvas. Editing SVG images is supported by SVG-edit ${ }^{8}$ with some predefined shapes which are commonly used in presentations. For logical structuring of presentations, SlideWiki utilizes a tree structure in which users can append new or existing slides/decks and drag \& drop items for positioning. When creating presentation decks, users can assign appropriate tags as well as footer text, default theme/transition, abstract and additional meta-data to the deck.

Revision control is natively supported by the WikiApp data model. We just define rules and restrictions to increase the performance. There are different circumstances in SlideWiki for which new slide or deck revisions have to be created. For decks, however, the situation is slightly more complicated, since we wanted to avoid an uncontrolled proliferation of deck revisions. This would, however, happen due to the fact, that every change of a slide would also trigger the creation of a new deck revision for all the decks the slide is a part of. Hence, we follow a

\footnotetext{
${ }^{6}$ http://slidewiki.org

${ }^{7} \mathrm{http}: / /$ aloha-editor.org/

http://code.google.com/p/svg-edit/
} 
PAPER

CROWDLEARN: CROWD-SOURCING THE CREATION OF HIGHLY-STRUCTURED E-LEARNING CONTENT

TABLE I.

IMPLEMENTATION OF THE DIFFERENT ASPECTS OF COLLABORATIVE EDUCATIONAL CONTENT AUTHORING IN SLIDEWIKI

\begin{tabular}{|c|c|c|c|}
\hline \multirow{4}{*}{ Category } & Feature & Support in SlideWiki & Approach (if supported) \\
\hline \multirow{4}{*}{$\begin{array}{c}\text { Support of col- } \\
\text { laboration }\end{array}$} & Collaborative content authoring & yes & Wiki-based \\
\cline { 2 - 4 } & Collaborative metadata authoring & yes & Wiki-based \\
\cline { 2 - 4 } & Colaborative assessment items authoring & yes & Wiki-based \\
\cline { 2 - 4 } & Collaboration for quality assurance & no & Collaborative annotation \\
\cline { 2 - 4 } & Collaborative content categorization & no & CoSMEC \\
\hline \multirow{3}{*}{$\begin{array}{c}\text { Support of reuse } \\
\text { and re-purpose }\end{array}$} & Collaborative content personalization & yes & no \\
\cline { 2 - 4 } & Collaborative content localization & yes & WikiApp \\
\hline \multirow{4}{*}{$\begin{array}{c}\text { Support of social } \\
\text { aspects }\end{array}$} & Advanced search & yes & WikiApp \\
\cline { 2 - 4 } & Content remixing & yes & Social networking \\
\cline { 2 - 4 } & Content organization/structuring & yes & Social networking \\
\cline { 2 - 4 } & Content negotiation & no & yes \\
\hline
\end{tabular}

more retentive strategy. We identified three situations which have to cause the creation of new revisions:

- The user specifically requests to create a new deck revision.

- The content of a deck is modified (e.g. slide order is changed, change in slides content, adding or deleting slides to/from the deck, replacing a deck content with new content, etc.) by a user which is neither the owner of a deck nor a member of the deck's editor group.

- The content of a deck is modified by the owner of a deck but the deck is used somewhere else.

In addition, when creating a new deck revision, we always need to recursively spread the change into the parent decks and create new revisions for them if necessary.

\section{B. Collaborative authoring of assessment items}

SlideWiki supports the creation of questions and selfassessment tests based on slide material. Each question has to be assigned to at least one slide. Important note here, that the question is assigned not to the slide revision, but to slide itself. Thus, when a new slide revision appears, it continues to include all the list of previously assigned questions. Questions can be combined into tests. The automatically created tests include the last question revisions from all the slides within the current deck revision. Manually created tests present a collection of chosen questions and currently cannot be manipulated as objects (cf. fig:screenshot, image 2). Thus, in our implementation only questions and answers have to be placed under the version control. However, their structure is trivial and the logic of creating their new revisions is intuitive. We just restricted the number of new revisions to be created similarly with the decks: changes made by the question owner do not trigger a new revision creation.

For now, only multiple-choice (and multiple-mark) question type is implemented, however in the future we plan to expand the list of supported types. To score the results the student (or the teacher) can choose one of five implemented algorithms, including our innovative balanced scoring one [17] .

Students can start a chosen test (manually created or automatically collected) in one of two possible modes: "learning" or "examination". In learning mode student can ask to show the slide, to which the question is assigned to remind the material, or simply show the correct answers (cf. Figure 2, image 3). Thus, student should not spend time to find the material. However, after the user asked to show her/him either the slide or correct answers she/he will not get any points for the question. In examination mode these features are disabled.

After choosing the mode the user can also set up the amount of questions (all, all the difficult or concrete amount) and the order to show them (random or increase the difficulty). As the amount of questions can differ for the same test, we show the test results as a percentage of the maximum points for exactly this selection of question.

Our architecture allowed us to implement module-based scoring. Each module of the assessment test presents a sub-deck of the presentation and is scored individually. Then, all the "parent" modules are scored as a sum of "children" points and finally the whole test is scored as a sum of all the points for all the modules (cf. Figure 2, image 4).

\section{Collaborative content categorization}

The issue of collaborative content categorization is well addressed by recent research in the field. In SlideWiki we implement a simple solution, namely social tagging. The users can add tags to slide decks and later search the content using tag clouds. In the future we plan to implement more sophisticated approaches, using for example: named entity recognition techniques and knowledge bases like DBpedia.

\section{Collaborative content localization}

The support of multilingual contentis implemented by adding the translatedInto and its inverse translatedFrom 
PAPER

CROWDLEARN: CROWD-SOURCING THE CREATION OF HIGHLY-STRUCTURED E-LEARNING CONTENT

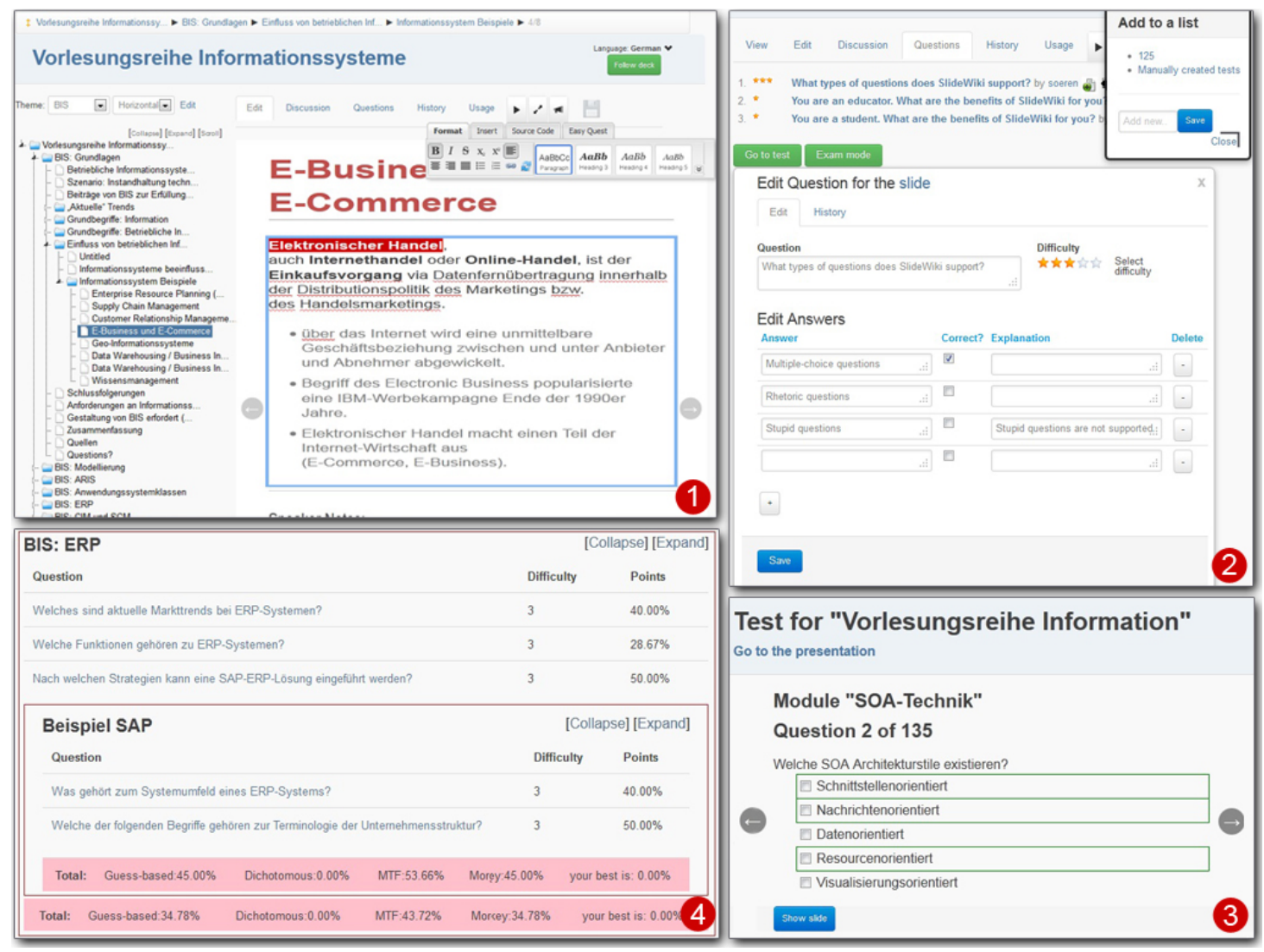

Figure 2. Four screenshots of SlideWiki features. 1 - Tree structure of the presentation, inline WYSIWYG editor; $\mathbf{2}$ - Editing of a question and manual assigning to a test using lists; $\mathbf{3}$ - Question in learning mode with correct answers displayed; $\mathbf{4}$ - Module-based scoring of an assessment test.

relations to the WikiApp data model. In order to provide the synchronisation of the content versions we introduce the paradigm of co-evolution of multilingual content, that means the ability to update a translation to the current state of the source object and vice versa. Details of the concept are presented in [16], in the current paper we briefly summarize the implementation of the concept in SlideWiki.

The implementation of co-evolution of source object content and its translations supposes the implementation of three operations: initial translation, synchronization and merging of the revisions. In this subsection we describe an example implementation of these operations in SlideWiki.

\section{1) Translation.}

Our architecture allowed us to implement a semiautomatic translation feature backed by the Google Translate service. After the translation into one of 91 supported languages, the presentation can be edited (localized) independently from the original one.

\section{2) Synchronization.}

To enable synchronization of original and translated versions, every further revision of translated objects inherits the link to the source revision (see v2.1, Figure 3).

The users are able to compare v1.0 and v1.1 and decide, either they want to redo the translation (scenario 1 at Figure 3 ) or they want to update the content manually (scenario 2).

\section{3) Merging the revisions.}

SlideWiki implements the revision control in accordance with the WikiApp data model. However, we defined rules and restrictions to increase the performance. We wanted to avoid an uncontrolled proliferation of deck revisions. However, this would happen due to the fact that every change in a slide would also trigger the creation of a new deck revision for all the decks that slide is a part of. In addition, when creating a new deck revision, we always need to recursively spread the change into the parent decks and create new revisions for them if necessary. To deal with this issue, we introduced the content owner and member of editor group roles. If the changes are made by a user belonging to one of these two roles, the creation of a new deck revision is not triggered (the new slide revision however is created). As we allow the owner of a deck revision to change it without the creation of a new revision, it was an important issue whether we should allow the multiple translation of the same revision into the same language or not. We decided to allow it, however, this led to the situation that we would get several identical presentations with content of bad quality, since it was translated automatically and not edited manually. However, we could not disable the multiple translations, because in that case it would be impossible for example to get translations of new slides if they were added by the owner. Thus, merging the revisions became the crucial operation, not only for merging back-translation with the source, but also for merging multiple translations in the same language. 


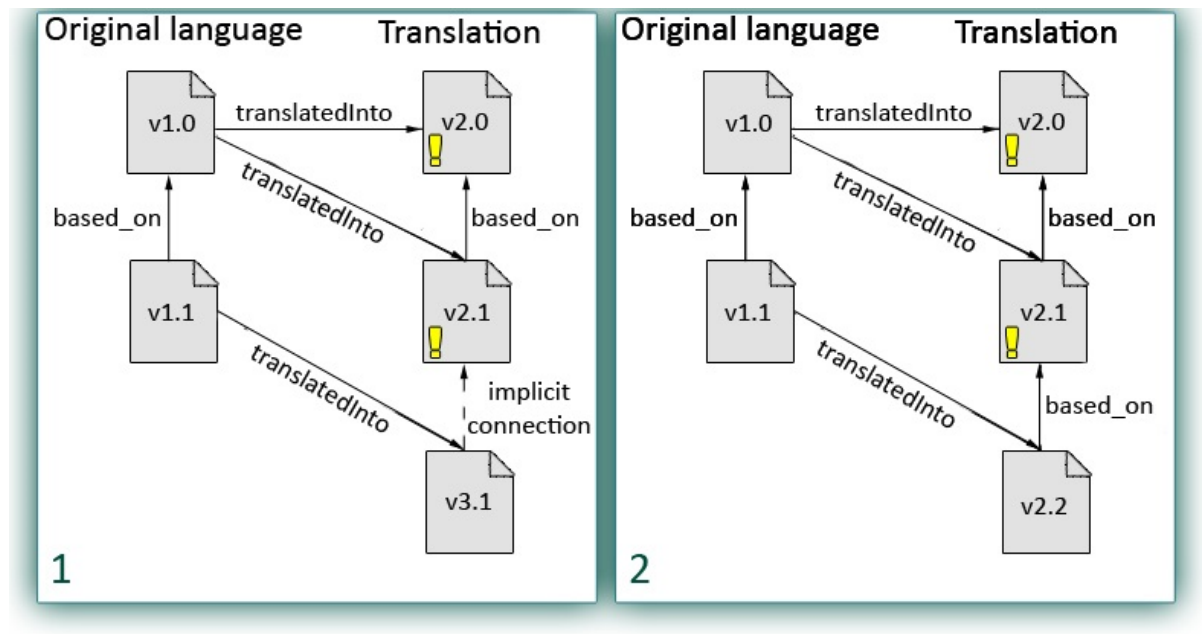

Figure 3. Two scenarios of content synchronization between translation and source: 1 - automatic; 2 - manual synchronization

\section{E. Content remixing and structuring}

The content remixing and structuring is supported by WikiApp data model. It adds some additional formalisms in order to make users as well as ownership, part-of and based-on relationships first-class citizens of the data model. A set of content objects connected by part-of relations can e manipulated in exactly the same manner as an individual non-structured object. For example, users can copy and paste parts of a deck to multiple new decks, remove parts of their own deck versions, without raising conflicts between different content versions.

\section{F. Content negotiation and user awareness}

The content negotiation and user awareness aspects are implemented in SlideWiki based on social networking mechanisms. Each content object, i.e. slide, deck or question, has its own comments thread. The comments are copied from version to version together with the content they refer to. This allows the users to avoid discussing the same issues repeatedly.

The user awareness is supported by the content following and graphic notification systems. When a user follows a deck, she starts to be informed about all new versions of the deck, subdecks and individual slides it contains. When a user follows another user, she gets notified of all new content the other user creates.

\section{G. User engagement}

In order to motivate users to contribute to the SlideWiki content we use the gamification strategy, namely the digital badges. This strategy suggests that users who achieved a certain level in a certain activity gets a graphically represented prove of that, which can be shared through a number of channels. In SlideWiki the activities for which a badge can be granted include content contribution, content translation and self-assessment with multiple-choice tests.

The content contribution activity is measured by a number of authored slides and the badges are granted when 100, 500 and 1000 slides are authored by the user. The content translation activity badge is granted when 100,500 or 1000 automatically translated slides were revised by a user. The self-assessment activity badge does not have a gradation and is granted when a user achieves $100 \%$ of correct answers in a test with more than 10 questions not authored by the user. All badges are accompa- nyed by metadata, which shows all necessary details about the badge: the platform issued it (in our case SlideWiki), the criteria for issue, the date of issue, a link to the prove if possible. To manage and store the badges we provide the export of them into Mozila Backpack ${ }^{9}$. Using the backpack users can revise the badges achieved and export it to a number of social networking platforms supporting digital badges. One of those platforms is SlideWiki itself, where the badges can be shown in the user profile, when chosen to do so in the backpack.

\section{Evaluation}

To evaluate the real-life usability of SlideWiki, we used it for accompanying an information systems lecture at Chemnitz Technical University. We structured the slides within the lecture series and added questions for student self-assessment before the final exam. We informed them about the different e-learning features of SlideWiki, in particular, how to prepare for the exam using SlideWiki. The experiment was not obligatory but students actively contributed by creating additional questions and fixing mistakes. The experiment was announced to 30 students of the second year and 28 of them registered at SlideWiki.

The students were working with SlideWiki for several weeks, and we collected the statistics for that period. During that period, they created 252 new slide revisions which some of them were totally new slides, others were improved versions of the original lecture slides. Originally the whole course had 130 questions, and students changed 13 of them, fixing the typos or adding additional distractors to multiple choice questions. In total, students performed 287 self-assessment tests. The majority of these used the automatically and randomly created tests covering the whole course material. 20 tests included only difficult questions, 2 asked to show the questions with increasing difficulty. This showed us that the students liked the diversity of test organization. Students also liked the possibility to limit the number of questions - 80 attempts were made with such a setting. 8 students reached the $100 \%$ result for the whole course. On average, it took them 6 attempts before they succeeded.

After the experiment we can claim, that more active SlideWiki users received better marks on the real exami-

\footnotetext{
${ }^{9}$ https://backpack.openbadges.org/
} 
nation. It shows that SlideWiki not only allows students to prepare for the examinations, but also engages them in active participation that helps to improve the quality of the learning.

Another important aspect we wanted to evaluate is how actively will the students use the multilinguality support of SlideWiki. In order to do this we collected statistics on the usage of the translation tool as well as statistics on the multilingual content it produced.

Figure 4 presents the distribution between original and translated versions in relation to the total number of content objects. As the WikiApp data model does not enable deletion or update of the content, the graphs can be viewed as time trends. The blue line shows an example moment in time, when the SlideWiki database consisted of 16321 slides overall. The graphic shows that $78 \%$ of the slides at the moment were in their original language, $22 \%$ were translations and $5 \%$ of total number of slides were revised after translation. At the same moment, about $35 \%$ of decks were translations. Thus, the percentage of translated decks increases faster than the same for slides. This means that the presentations consisting of less than average number of slides are being translated more often. This can be due to the fact that users want to try the feature before using it on large decks. However, the assumption needs further investigation.

According to the statistics, the percentage of content created by translation has a strongly increasing trend. We predict the percentage of translations will soon prevail over the percentage of source objects. From one perspective, the prevalence means decreasing the production costs and a large diversity of languages available. However, from another perspective, it causes the reduction of average content quality, as refining the translation needs time and human resources. This is illustrated by the decreasing percentage of revised slides in compartment with translated ones. The solution is in additional user motivation to put effort in refining the concrete translations according to their knowledge in both domain and languages.

The diagrams on the Figure 5 show the language distributions for decks, slides and visitors (according to Google analytics). Only unique visitors are counted. Due to its large percentage, we excluded English language from the resulting diagrams to increase readability. The statistics show the visible correlation between number of content objects available in a concrete language and number of visitors speaking the language (for the most of languages). The results look promising, as they prove the involvment of non- English-speakers into the global e-learning community activity. Especially promising is the fact that more than $13 \%$ of overall visitors belong to developing countries and regions (mostly Eastern Europe and Russia, Turkey, Arabic-speaking countries, Thailand). We believe that this percentage will grow with increasing the popularity of the source.
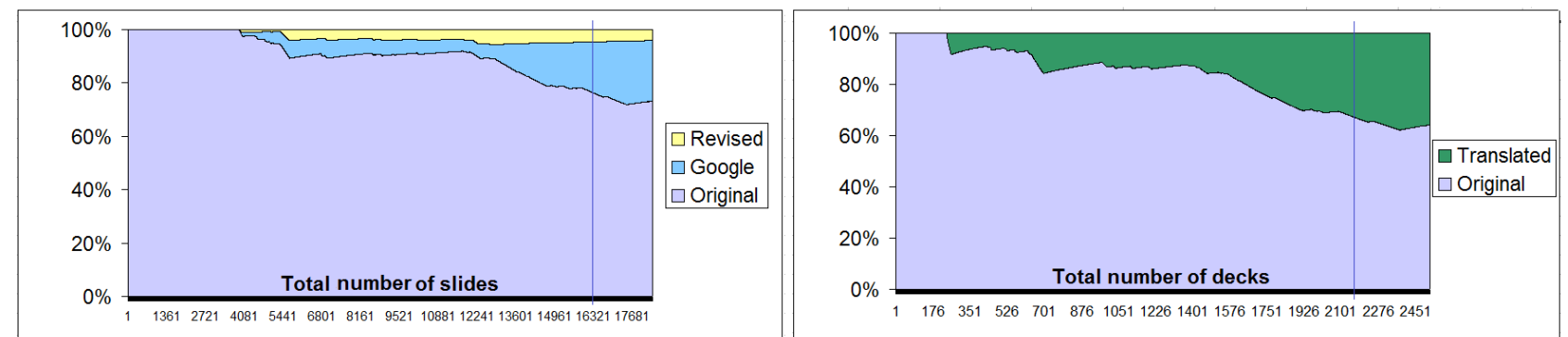

Figure 4. Percentage distribution between original and translated versions vs. total number of content objects for slides and decks. Blue line shows an example time moment, discussed in the paragraph above

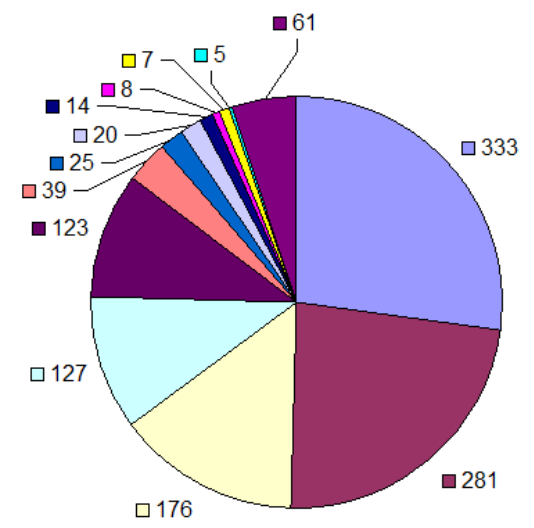

a. Decks

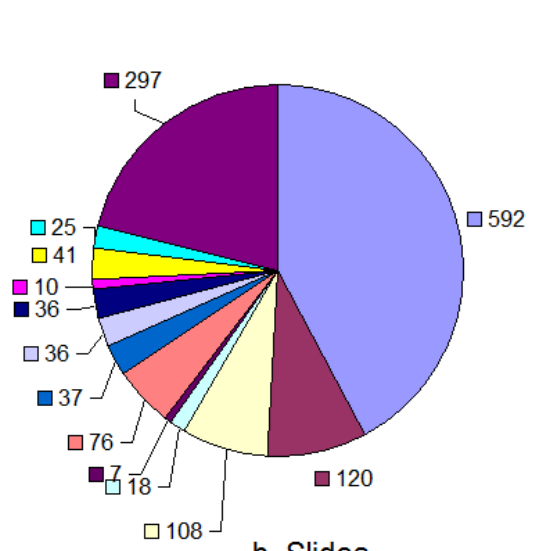

b. Slides

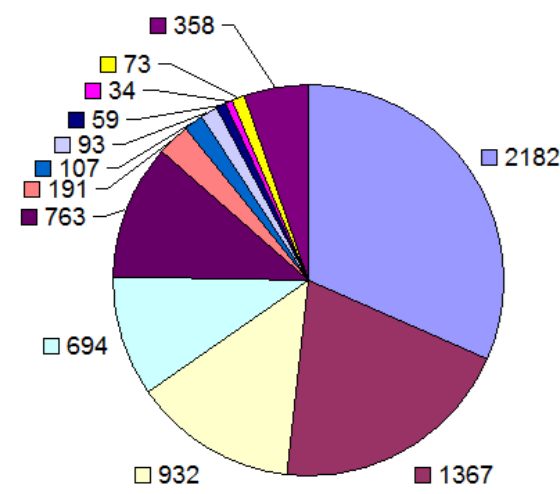

c. Visitors

$\square$ de $\square$ fr $\square$ ru $\square$ th $\square$ fa $\square$ es $\square$ pt $\square$ ar $\square$ it $\square$ el $\square$ pl $\square$ tr $\square$ others

Figure 5. Distribution of languages for content objects and new visitors 


\section{CONCLUSIONS AND FUTURE WORK}

In this paper we presented the CrowdLearn concept that applies collaborative authoring and crowd-sourcing techniques to the creation of (semi-) structured elearning content. The concept is based on the SCORM concepts and uses the novel WikiApp data model to organize the content closely aligned with the standard. We implemented and evaluated the concept with SlideWiki - a social web e-learning application targeting slide presentations and e-assessments. While the evaluation results were promising, we still need to extend the concept in the future to address the requirements requested by users.

Beside the usability improvements, our first direction for future work is to implement a completely SCORMcompliant LMS and authoring tool, based on the SlideWiki. This will allow us to exchange the content with other SCORM-compliant LMSs.

In a real e-learning scenario, learners come from different environments, have different ages and educational backgrounds. These heterogeneities in user profiles are crucial to be addressed when enhancing the CrowdLearn concept. New approaches should provide the possibility to personalize the learning process. Thus, our second direction is providing the personalized content based on initial user assessments. The third direction for the future work is to support the annotation of learning objects using standard metadata schemes. We aim to implement the $L R M I^{10}$ metadata schemes to facilitate end-user search and discovery of educational resources.

\section{REFERENCES}

[1] SCORM 2004 4th Edition Specification. Technical report, ADL, 2011.

[2] SCORM Users guide for programmers. Technical report, ADL, 2011.

[3] Auer, S., Dietzold, S., Lehmann, J., Hellmann, S., and Aumueller, D. (2009). „Triplify: Light-weight linked data publication from relational databases", WWW2009, Spain.

[4] Barriocanal, E. G., Sicilia, M.-Á., Alonso, S. S., and Lytras, M. D. "Semantic annotation of video fragments as learning objects," Interactive Learning Environments, 19(1):25-44, 2011 http://dx.doi.org/10.1080/10494820.2011.528879

[5] Devedzic, V. "Semantic Web and Education (Integrated Series in Information Systems)," Springer, Secaucus, NJ, USA, 2006

[6] Haake, A., Lukosch, S., and Schümmer, T. "Wiki-templates: adding structure support to wikis on demand," International Symposium on Wikis, pp 41-51, ACM, 2005 http://dx.doi.org/10.1145/1104973.1104978
[7] Howe, J. " The rise of crowdsourcing," Wired Magazine, 14(6), 2006

[8] Jones, E. "Implications of scorm and emer ging e-learning standards on engineering education," ASEE Gulf-Southwest Annual Conference, 2002

[9] Kuo, Y.-H., Kinshuk, Q. T., Huang, Y.-M., Liu, T.-C., and Chang, M. "Collaborative creation of authentic examples with location for u-learning," International Conference e-Learning, pp. 16 - 20. IADIS, 2008.

[10] Leuf, B. and Cunningham, W. "The Wiki way: quick collaboration on the Web," Addison-Wesley, London, 2001.

[11] Lewis, J. and Sauro, J. "The Factor Structure of the System Usability Scale," Human Centered Design, volume 5619 of LNCS, chapter 12, pp. 94-103. Springer, 2009. http://dx.doi.org/10.1007/978-3-642-02806-9_12

[12] Matthes, F., Neubert, C., and Steinhoff, A. "Hybrid wikis: Empowering users to collaboratively structure information," ICSOFT (1), pp. 250-259, 2011.

[13] Pedreira, N., Salgueiro, J. R. M., and Carballo, M. M. "Elearning in new technologies," Encyclopedia of Artificial Intelligence, pages 532-535. IGI Global, 2011.

[14] Richards, D. "A social software/web 2.0 approach to collaborative knowledge engineering," Inf. Sci., 179(15):2515-2523, 2009. http://dx.doi.org/10.1016/j.ins.2009.01.031

[15] Tarasowa, D., Khalili, A., Auer, S. and Unbehauen, J. "CrowdLearn: Crowd-sourcing the Creation of Highlystructured e-Learning Content,", CSEDU, 2013.

[16] Tarasowa, D., Auer, S., Khalili, A. and Unbehauen, J. "Crowdsourcing (semantically) Structured Multilingual Educational Content (CoSMEC)," Open Praxis 6(2), pp. 159-170, 2014. http://dx.doi.org/10.5944/openpraxis.6.2.117

[17] Tarasowa, D. and A., Auer, S. "Balanced scoring method for multiple-mark questions,", CSEDU, 2013.

[18] Tarasowa, D. and Auer, S. "Collaborative Authoring of OpenCourseWare: a state-of-art analysis", unpublished.

[19] Wang, X., Love, P. E., Klinc, R., Kim, M. J., and Davis, P. R. "Integration of e - learning 2.0 with web 2.0," ITcon - Special Issue eLearning 2.0: Web 2.0-based social learning in built environment, 17:387-396, 2011.

\section{AUTHORS}

D. Tarasowa is with Rheinische FriedrichWilhelms-Universität Bonn, Germany.

A. Khalili is with Vrije Universiteit Amsterdam, The Netherlands.

S. Auer is with Rheinische Friedrich-WilhelmsUniversität Bonn, Germany.

This article is an extended and modified version of a paper presented at the 5th International Conference on Computer Supported Education (CSEDU 2015), 23-25 May 2015, Lisbon, Portugal. Submitted 09 August 2015. Published as resubmitted by the authors 10 October 2015.

\footnotetext{
${ }^{10}$ Learning Resource Metadata Initiative: www.lrmi.net/
} 